\title{
Atomic Structure of the Polarization Modulations in Perovskite Antiferroelectrics
}

Tao Ma ${ }^{1}$, Binzhi Liu ${ }^{2}$, Zhongming Fan ${ }^{2}$, Tae-Hoon Kim ${ }^{3}$, Xiaoli Tan $^{2}$ and Lin Zhou ${ }^{3}$

${ }^{1}$ University of Michigan, Ann Arbor, Michigan, United States, ${ }^{2}$ Iowa State University, Ames, Iowa, United States, ${ }^{3}$ Ames Laboratory, Ames, Iowa, United States

Antiferroelectric (AFE) and ferroelectric (FE) materials have played indispensable roles in modern industry, such as electricity storage, microscale cooling, precise positioning, and nonvolatile memories. Their functions in these applications are rooted from the spontaneous dipoles within unit cells manifested in the form of off-center displacements of cations and anions. At the length-scale of domains, dipoles are believed in an antiparallel arrangement in neighboring stripes in AFE domains, so that the macroscopic polarization is fully compensated.[1,2]

$\mathrm{PbZrO}_{3}$ is the most widely studied AFEs, which crystallizes in a distorted perovskite structure with antiparallel $\mathrm{Pb}$-cation displacements in the commensurate modulations (CMs) of 1/4 \{110 $\}_{c}$, i.e., four layers of its pseudocubic $\{110\}_{c}$ plane. As shown in Figure 1a,b the antiparallel arrangement of the $\mathrm{Pb}$ cation displacements in pure $\mathrm{PbZrO}_{3}$ is directly verified by the mapping of $\mathrm{Pb}$-displacement vectors on a high-angle-annular-dark-field (HAADF) scanning transmission electron microscopy (STEM) image. Because critical electric field $\left(\mathrm{E}_{\mathrm{F}}\right)$ in $\mathrm{PbZrO}_{3}$ at room temperature exceeds its dielectric strength, $\mathrm{Sn}$ and $\mathrm{Ti}$, in conjunction with a small amount of $\mathrm{Nb}$ or $\mathrm{La}$, are added to reduce $\mathrm{E}_{\mathrm{F}}$ for practical applications. The reduction in $E_{F}$ is accompanied with the formation of $1 / n\{110\}_{c}$ ( $n$ is non-integer, typically between 6 and 8) incommensurate modulations (ICMs), which manifest themselves as fine fringes in diffraction-contrast transmission electron microscopy (TEM) images and satellite spots in reciprocal space (Figure 1c, d). Despite of the stochastic thickness of ICMs, which may contain 4-8 layers of $\{110\}_{\mathrm{c}}$, the dipoles in adjacent stripes were assumed to be antiparallel to ensure the overall polarization in AFE domains remain compensated.[2]

Here we demonstrate that this classic view of the dipole arrangement in ICMs is incorrect. With direct cation displacement mapping in a series of $\mathrm{PbZrO} 3$-based compositions ( $\mathrm{Pb} 0.99 \mathrm{Nb}_{0.02}\left[\left(\mathrm{Zr}_{0.57} \mathrm{Sn}_{0.43}\right)_{1-}\right.$ y $\left.\mathrm{Ti}_{\mathrm{y}}\right]_{0.98} \mathrm{O}_{3}$ ), we reveal that the $\mathrm{Pb}$ displacements in adjacent stripes of ICMs are frequently uncompensated. They are either antiparallel but with different magnitudes along opposing directions, or in a nearly orthogonal arrangement, i.e., these previously thought fully-compensated AFE domains in fact bear a net polarization (Fig. 1d). Moreover, with in situ electric-biasing TEM experiments, we demonstrate that these polar AFE domains transition into FE domains under applied electric fields and resume their original configuration upon removal of the field, leading to the AFE-like double P-E hysteresis loops. Our discovery not only revises the atomistic understanding of ICMs in perovskite ferroics, but also sheds light on establishing the structure-property relationship in many other multifunctional materials.[3,4] 


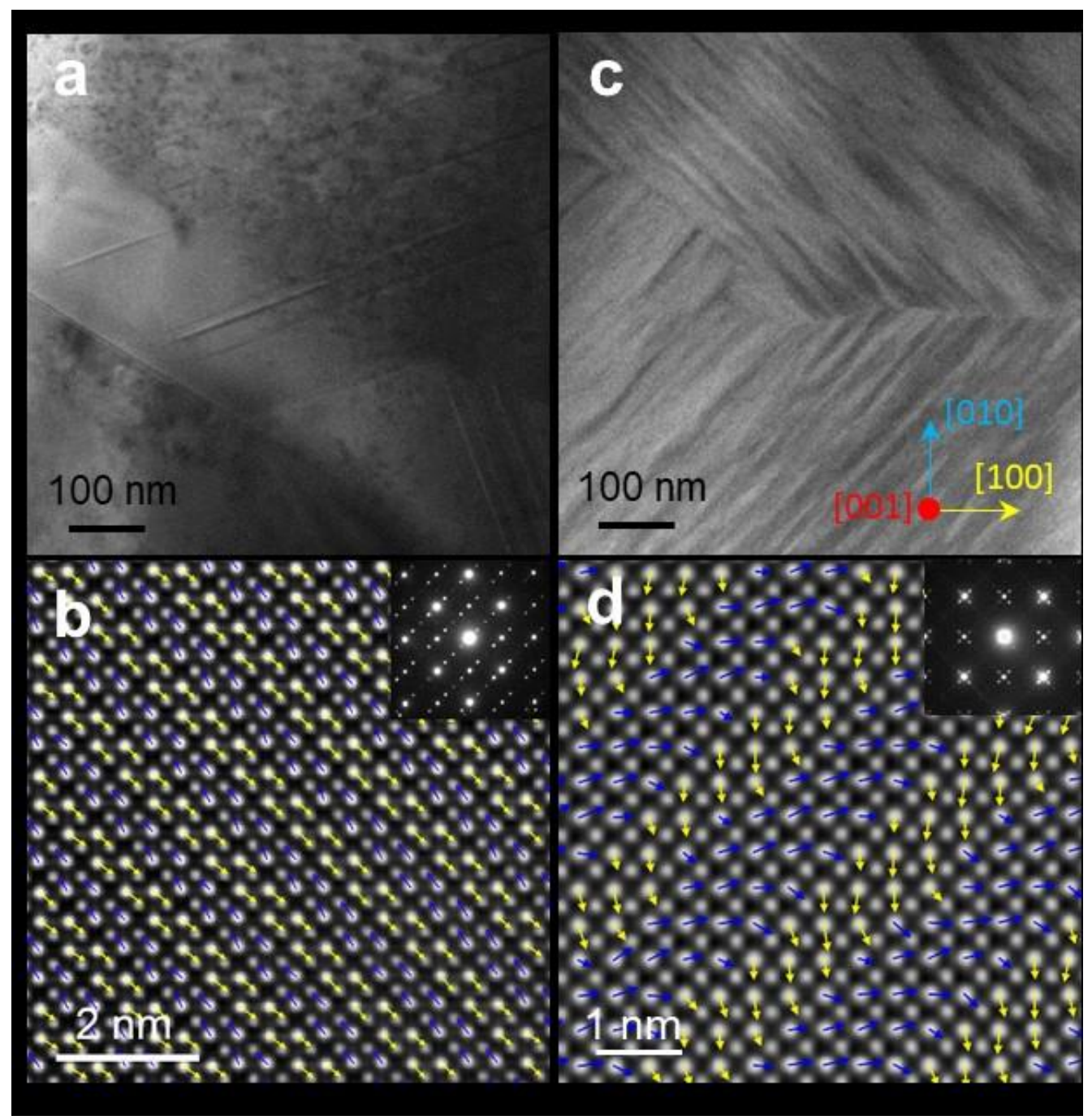

Figure 1. (a) Bright-field TEM image and (b) Pb-displacement vector map overlaid on the HAADFSTEM image of PbZrO3. (c) Bright-field TEM image and (d) Pb-displacement vector maps overlaid on the HAADF-STEM image from the bottom domain of $(\mathrm{Pb} 0.99 \mathrm{Nb} 0.02[(\mathrm{Zr} 0.57 \mathrm{Sn} 0.43) 0.94 \mathrm{Ti0} .06] 0.98 \mathrm{O} 3)$. The inset is corresponding SAED along [001]c zoneaxis.

\section{References}

[1] Kittel, C. Theory of antiferroelectric crystals. Phys. Rev. 82, (1951) 729-732.

[2] X. Tan, C. Ma, J. Frederick, S. Beckman, and K. G. Webber, The antiferroelectric $\leftrightarrow$ ferroelectric phase transition in lead-containing and lead-free perovskite ceramics. J. Am. Ceram. Soc. 94, (2011) 4091-4107.

[3] Tao Ma, Zhongming Fan, Bin Xu, Tae-Hoon Kim, Laurent Bellaiche, Matthew J. Kramer, Xiaoli Tan, and Lin Zhou, Physical Review Letters, 123 (2019) 217602.

[4] This work was supported by the National Science Foundation (NSF) through Grant DMR-1700014. All electron microscopy work was performed at the Sensitive Instrument Facility at Ames Laboratory, which is operated for the U.S. DOE by Iowa State University under Contract No. DE-AC02-07CH11358. 